\title{
Framing the Watercolour and Gouache Study Collection from VSAR-1060: Studio Practice and Ideas/Image
}

\author{
Catherine Heard \\ University of Windsor
}

VSAR-1060 Studio Practice and Ideas/Image is a first-year course in the School of Creative Arts at the University of Windsor that introduces students to the fundamental concepts of 2-dimensional image-making. This assignment was designed to teach the complexities and nuances of colour mixing, and to reinforce students' understanding of the elements of art and the principles of design.

After viewing a short video about David Batchelor's colour sculptures made of found objects, students were directed to apply their knowledge of compositional concepts and colour relationships to create small abstract sculptures using materials they had brought from home. These sculptures were crafted quickly using a glue gun. Students made backdrops for their sculptures with colorful paper or fabric, then used desk lamps and coloured gels to light their sculptures creatively. Only after completing this work did students learn their creations would be the inspiration for a series of studies in watercolour and gouache.

The students' first three studies were made with watercolours, using a limited palette of Winsor Blue, Winsor Yellow, and Permanent Rose to replicate the CMY (cyan/magenta/yellow) mixing system used in commercial printing. To help students focus on compositional decisions and colour mixing, they were encouraged to work on the three paintings simultaneously. This method also made the best use of their studio time, as students could be working on one painting while washes on their other works-in-progress were drying. For this assignment, Winsor and Newton Professional Watercolours were chosen so that students could experience the difference that working with artist quality paints could make to their work. These paints have a high pigment load, which produces pure, vibrant colours.

Next, students developed three gouache studies inspired by Michael Wilcox's Blue and Yellow Don't Make Green (1987), which explains that every primary colour has an underlying warm or cool cast that affects the way secondary colour mixes look. Wilcox's system of colour mixing helps the student artist predict if a colour will be intense or muted, pure or muddy. For this exercise students used a palette composed of Turner Design Gouache Permanent Lemon, Permanent Yellow Deep, Permanent Scarlet, Rose, Ultramarine Blue, and Sky Blue as their primary colours. In addition, they explored warm and cool blacks using Jet Black and Lamp Black; and learned about colour strength (opacity) by comparing Titanium White and Mixing White (Zinc White). As in their watercolour exercises, students worked on three paintings simultaneously to facilitate comparison and to allow time for drying. 
One of the benefits of students producing a series of three small works in each medium is that they are less invested in individual works, making it easier for them to experiment and take risks. Additionally, when students create serial works, it tends to diffuse their natural inclination to compare their works to those of their peers. Instead, students can identify which of their own works was most successful and are encouraged to recall and analyze what strategies they employed in that work. Translating their sculptures into two series of works executed in different mediatransparent watercolour and opaque gouache-encouraged students to consider which medium best suited their personal approach to image making. Some students were delighted by the surprises that occurred when watercolours were allowed to flow and appreciated the nuanced layers of colour they could create in this medium; while others preferred working in gouache, which permits precise control and hard edge compositions. Discovering their strengths and personal stylistic preferences through process-oriented experimentation builds students' confidence and prepares them to progress to second-year courses, which will further develop their emerging artistic voices.

\section{References}

Wilcox, M. (1987). Blue and yellow don't make green: Or how to mix the colour you want - every time. Perth, Australia: Artways. 